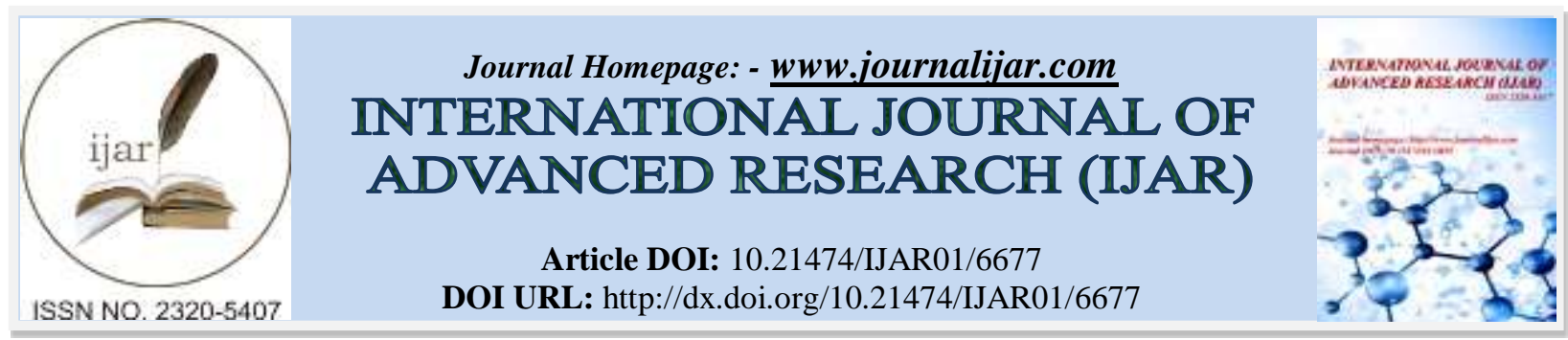

RESEARCH ARTICLE

\title{
EVALUATION OF THE EFFECT OF PLATELET-RICH FIBRIN ON PALATAL DONOR AREA PAIN AFTER HARVESTING A FREE GINGIVAL GRAFT.
}

\author{
"Devrim Deniz Üner ${ }^{1}$ and Bozan Serhat Izol ${ }^{2}$. \\ 1. Harran University Faculty of Dentistry Department of Periodontology. \\ 2. Dicle University Faculty of Dentistry Department of Periodontology.
}

\section{Manuscript Info}

Manuscript History

Received: 05 January 2018

Final Accepted: 07 February 2018

Published: March 2018

Keywords:-

palatal bandage, free gingival graft, platelet-rich fibrin, palatal donor area.

\begin{abstract}
The aim of this study was to evaluate the early healing of palatal wounds after free gingival grafting (FGG) procedures by comparing wounds dressed with platelet-rich fibrin membranes (PRFs) and wounds treated without any dressing material. Twenty patients with Miller class I-II gingival recession requiring a root-coverage procedure were selected for treatment with an FGG technique and were randomly assigned to two groups. The size of the graft was standardized to 10$10 \mathrm{~mm}$, and the thickness ranged from $1 \mathrm{~mm}-1.5 \mathrm{~mm}$. The subjective parameter pain was recorded on the second and seventh days following surgery.

The mean VAS scores for pain in the test group varied from 2.3 and 0.58 , respectively, on the second and seventh days following surgery. The VAS scores for pain in the control group were 6.21 and 2.63, respectively, on the second and seventh days after the procedure.

Statistically significant differences were found between the VAS scores of the test and control groups. The high VAS scores in the control group indicate that the use of PRF membranes as the palatal bandages for wound closure at the palatal donor site following FGG procedures is important for ensuring patient comfort.
\end{abstract}

Copy Right, IJAR, 2018,. All rights reserved.

\section{Introduction:-}

The free gingival graft (FGG) procedure for the treatment of mucogingival problems, gingival recessions, and loss of attached gingiva (Echeverria, Montero et al. 2002) was first described by Björn in 1963. The keratinized gingiva of the hard palate is the most accessible site from which to obtain free soft-tissue grafts for use in various types of plastic periodontal surgery (Cortellini and Pini Prato 2012). For anatomical reasons, the palatal mucosa in the premolar region is the ideal area for graft placement (Reiser, Bruno et al. 1996). The FGG technique is widely used in the periodontal surgical treatment of mucogingival problems (Dorfman, Kennedy et al. 1982). The postoperative complications of FGG are associated with an open secondary wound site and include pain, postsurgical bleeding, and necrosis (Brasher, Rees et al. 1975, Zucchelli, Mele et al. 2010). Numerous studies have been conducted to find ways in which to reduce postoperative complications. These studies have confirmed the effective use of hemostatic agents, ozonated oil, herbal products, antibacterial agents, bioactive materials, low-level laser therapy, and platelet concentrates (Keceli, Aylikci et al. 2015). In addition, after periodontal surgery, various dressing materials have been used to protect the wound surface against external factors (Farnoush 1978). Conventional periodontal dressing materials provide an inert mechanical barrier and thus assist healing by preventing external influences on the wound 
area (Rubinoff, Greener et al. 1986). Wound healing in the palatal region after FGG procedures is identical to that of the oral mucosa. During wound-healing fibroblast proliferation, collagen synthesis and accumulation, revascularization, and wound contraction is observed (Fahimipour, Houshmand et al. 2016). Platelet-rich fibrin (PRF), a second-generation platelet concentrate, was first produced in France by Choukroun et al. in 2001 (Choukroun, Adda et al. 2000). Three keys to wound healing and soft tissue maturation are angiogenesis, epithelial cover, and immunity. PRF, which is easily obtained and does not require any biochemical substances, is a natural fibrin-based biomaterial that can be used to guide the development of microvascularization and the migration of epithelial cells to the surface. For this reason, PRF membranes can be used to protect open wounds and to accelerate healing (Choukroun, Diss et al. 2006). The Task Force on Taxonomy of the International Association for the Study of Pain (IASP) concluded that pain is "An unpleasant sensory and emotional experience associated with actual or potential tissue damage, or described regarding such damage" (Glick 2015). Pain, as it can be understood from the description, is a subjective feeling that cannot easily be measured using objective methods. However, different methods of pain measurement have been developed, such as the verbal analog scale and the visual analog scale. The VAS consists of a 10-cm line, with the left-hand side labeled "no pain" and the right-hand side labeled "most intense pain imaginable" (or a similar descriptor) (Wynne, Ling et al. 2000).

This study aimed to evaluate palatal wound pain after FGG procedures by comparing the VAS pain levels of patients whose palatal wounds were dressed with PRF with those whose wounds were not treated with any dressing material.

\section{Materials and Methods:-}

Twenty healthy, non-smoking patients with gingival recession defect of the mandibular gingiva were selected for this study. Before the operation, initial periodontal treatment was administered to all patients, all of whom were reevaluated 3 months later. Verbal and written consents were obtained from the patients before the operations. After the recipient area was prepared with the patient under local anesthesia, a free gingival graft with an average size of $1 * 1 \mathrm{~cm}$ (to be standardized for the remaining wound surface area) was obtained from the palatal donor area. Each subject received a standardized FGG of the same dimensions. The FGG recipient area was sutured with $5 \backslash 0$ suture (Dogsan), and the palatal donor sites of 10 patients were covered with PRF membranes as palatal dressing (Figure 1). To obtain the PRF membranes, $5 \mathrm{ml}$ of venous blood was collected from the antecubital fossa of the patient and was immediately transferred to a sterile test tube. The blood was centrifuged at $3000 \mathrm{rpm}$ for 10 minutes, after which the PRFs were obtained. In each procedure, the PRF membrane was then shaped in two wet sterile gauzes and sutured to the donor site with $5 \backslash 0$ silk suture. The donor sites of the remaining 10 patients were left to heal conventionally (without PRF membranes) to compare their pain levels during the healing process with the patients whose wounds were treated with PRF membranes used as dressing. Postoperative instructions, including oral hygiene maintenance, antibiotics, and analgesics, were provided, and, on the tenth day following the procedure, the patients were evaluated and underwent suture removal. All the patients were prescribed the same analgesics and were recommended to use them twice a day. The VAS forms were given to patients to use in evaluating the degree of their pain after the procedure. The study used a visual analogic scale (VAS) ranging from 1 to 10, and the levels of outcomes were classified as "none to a minimum" "moderate" and very important/severe." On the second and seventh days following the FGG procedures, all patients were asked to assess their pain using the VAS. Appointments were made for suture removal for the PRF patients and control group postsurgical evaluations at 10 days after the procedure (Figure $2 \mathrm{a}-2 \mathrm{~b})$.

\section{Statistical Analysis:-}

Data were tabulated using Microsoft Excel (Microsoft, Redmond, WA, USA), and statistical analysis was performed using the mean, percentage, descriptives, and paired t-test; data homogeneity was tested using the Shapiro-Wilk test. SPSS Statistics version 20.0 (IBM Co. Armonk, NY, USA) was used to analyze the data. Statistics were considered significant if $\mathrm{P}<0.05$.

\section{Results:-}

The mean age of 20 female patients aged 22 to 32 years was calculated as $26.3 \pm 3.3$. The mean VAS score for pain in the patients with PRF dressings varied from $2.3 \pm 1.1$ to $0.58 \pm 0.4$, respectively, on the second and seventh days following the procedure. The mean VAS score of the patients without PRF dressings was 6.21 \pm 0.87 and 2.63 \pm 0.7 , respectively, on the second and seventh days after the procedure (Table 1). The analysis revealed that there was a 
statistically significant difference in the test group in which PRFs were used as palatal bandages compared to the group ( control group) left to healing secondary intention of VAS scores.

\section{Discussion:-}

Although the FGG procedure is considered to be predictable in achieving complete root coverage, the significant disadvantage of this procedure is the need to use the donor area for harvesting the graft and secondary healing intention (Camargo, Melnick et al. 2001). In the process of secondary healing, there may be several complications, including secondary bleeding, delayed epithelialization, and infections (Alkan and Inal 2008). Therefore after periodontal operations, various dressing materials are used to protect the wound surface from environmental conditions (Farnoush 1978). Although these dressing materials have demonstrated satisfactory clinical results, their ability to accelerate healing has been due largely to the mechanical protection of the wound area (Rubinoff, Greener et al. 1986). It is a series that includes inflammation, angiogenesis, and epithelization (Singer and Clark 1999), the three keys to healing and soft tissue maturation. The PRF membranes simultaneously support the development of the three keys to healing and soft tissue maturation, protect open wounds, and accelerate healing (Choukroun, Diss et al. 2006). It has been suggested that wound-related pain can have a negative impact on the wound healing (White 2008). Pain-related delays in wound healing are a particular concern for surgical patients, because healing of surgical wounds is a key outcome, and postsurgical pain is frequently undertreated (Bardiau, Taviaux et al. 2003). In recent studies, however, palatal donor sites covered with PRF membranes have been reported to heal considerably faster compared with sites allowed to heal conventionally (Aravindaksha, Batra et al. 2014, Kulkarni, Thomas et al. 2014, Gür, Ensari et al. 2017, Ozcan, Ucak et al. 2017). Therefore, in this study we used T-PRF as a palatal bandage to accelerate palatal wound healing and to decrease patient morbidity. Kulkarni et al. (2014) reported that sites where PRF was used showed complete wound closure within 14 days and that these patients reported less postoperative morbidity than patients whose wounds were not treated with PRF membranes (Kulkarni, Thomas et al. 2014). In a similar study, Shakir et al. reported that patients with PRF palatal dressings showed complete wound closure within 18 days and experienced less postoperative morbidity than a patient whose wounds were not treated with PRF membranes (Shakir, Bhasale et al. 2015). A study by Özcan et al. (2017) found that PRF membranes might provide significant benefits for wound healing parameters and a patient's postoperative course after palatal graft harvesting. In the present study, the VAS scores provided to us by patients who were treated with PRF palatal bandages were lower than the patients whose wounds had healed via conventional means, and wound healing occurred faster in the test group. For this reason, we concluded that PRF palatal bandages significantly accelerated palatal wound healing and reduced patient morbidity and pain.

\section{Conclusions:-}

The FGG procedure is one of the most commonly used methods for gingival augmentation. One of the most significant complications of this procedure is pain caused by healing via secondary intention at the palatal donor site after the operation. Clinicians have used different dressing materials to reduce pain in the palatal donor area. In this study, we used Choukroun's PRF as a dressing material in healing the palatal donor area and in reducing postoperative morbidity after harvesting an FGG. The present study indicates that the use of PRF membranes as palatal bandages could be an acceptable treatment for reducing postoperative complications following FGG procedures. To support the use of this technique, additional studies with larger numbers of patients are required.

\section{Conflict of Interest}

No potential conflict of interest relevant to this article was reported. 
Figure 1: Platelet-rich fibrin membrane secured to the palate.

Figure 2 a: View of donor area at 10 days post-op (without the use of PRF bandage)

Figure 2 b: View of donor area at 10 days post-op (with PRF bandage)

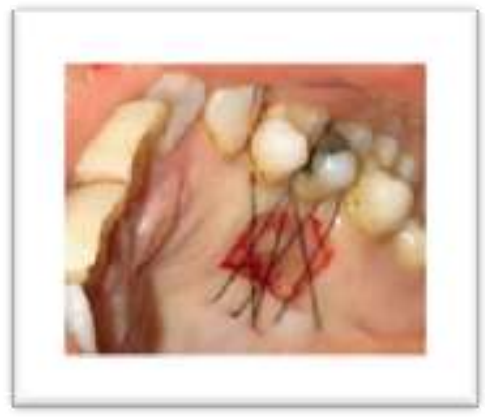

Figure 1

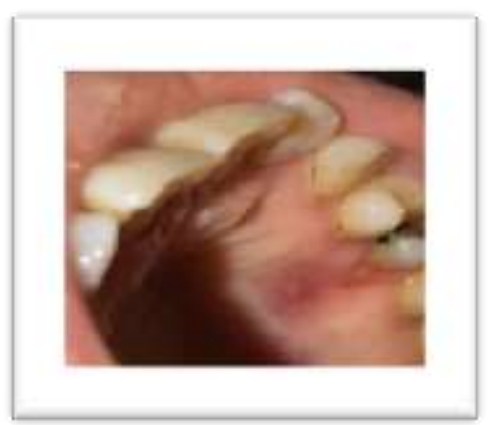

Figure 2a

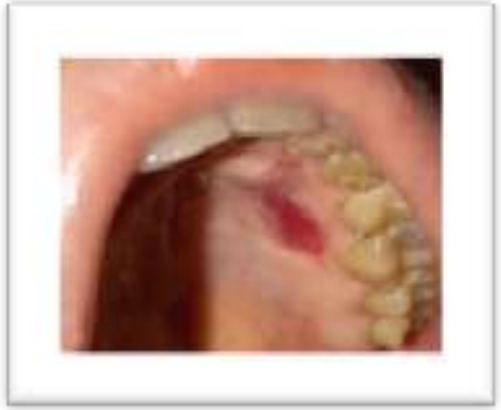

Figure 2 b

Table 1:- Comparison of VAS score for pain defined by the patients in post-op 2 and 7 days.

\begin{tabular}{|l|l|l|}
\hline \multirow{2}{*}{ 2 day after operation } & \multicolumn{2}{|c|}{ Vas Scores Means } \\
\cline { 2 - 3 } & Prf palatal bandage & Open wound healing \\
\hline 7 day after operation & $2,3 \pm 1,1$ & $6,21 \pm 0,87$ \\
\hline
\end{tabular}

\section{References:-}

1. Alkan, A. and S. Inal (2008). "Closure of palatal defects following excision of palatal pleomorphic adenomas." J Contemp Dent Pract 9(6): 99-107.

2. Aravindaksha, S. P., P. Batra, V. Sood, A. Kumar and G. Gupta (2014). "Use of platelet-rich fibrin membrane as a palatal bandage." Clinical Advances in Periodontics 4(4): 246-250.

3. Bardiau, F. M., N. F. Taviaux, A. Albert, J. G. Boogaerts and M. Stadler (2003). "An intervention study to enhance postoperative pain management." Anesthesia \& analgesia 96(1): 179-185.

4. Brasher, W. J., T. D. Rees and W. A. Boyce (1975). "Complications of free grafts of masticatory mucosa." Journal of periodontology 46(3): 133-138.

5. Camargo, P. M., P. R. Melnick and E. B. Kenney (2001). "The use of free gingival grafts for aesthetic purposes." Periodontology 2000 27(1): 72-96.

6. Choukroun, J., F. Adda, C. Schoeffer and A. Vervelle (2000). "PRF: an opportunity in perio-implantology (in French)." Implantodontie 42: 55-62.

7. Choukroun, J., A. Diss, A. Simonpieri, M. O. Girard, C. Schoeffler, S. L. Dohan, A. J. Dohan, J. Mouhyi and D. M. Dohan (2006). "Platelet-rich fibrin (PRF): a second-generation platelet concentrate. Part V: histologic evaluations of PRF effects on bone allograft maturation in sinus lift." Oral Surg Oral Med Oral Pathol Oral Radiol Endod 101(3): 299-303.

8. Cortellini, P. and G. Pini Prato (2012). "Coronally advanced flap and combination therapy for root coverage. Clinical strategies based on scientific evidence and clinical experience." Periodontology 2000 59(1): 158-184.

9. Dorfman, H. S., J. E. Kennedy and W. C. Bird (1982). "Longitudinal evaluation of free autogenous gingival grafts: a four year report." Journal of periodontology 53(6): 349-352.

10. Echeverria, J. J., M. Montero, D. Abad and C. Gay (2002). "Exostosis following a free gingival graft." Journal of clinical periodontology 29(5): 474-477.

11. Fahimipour, F., B. Houshmand, P. Alemi, M. Asnaashari, M. A. Tafti, F. Akhoundikharanagh, S. E. N. Farashah, M. Aminisharifabad, A. S. Korani and M. Mahdian (2016). "The effect of He-Ne and Ga-Al-As lasers on the healing of oral mucosa in diabetic mice." Journal of Photochemistry and Photobiology B: Biology 159: 149-154.

12. Farnoush, A. (1978). "Techniques for the protection and coverage of the donor sites in free soft tissue grafts." Journal of Periodontology 49(8): 403-405.

13. Glick, M. (2015). Burket's Oral Medicine, 12e, PMPH-USA.

14. Gür, Ö. E., N. Ensari and N. D. S. Y1lmaz (2017). "The effects of platelet-rich fibrin membrane on secondary healing after pleomorphic adenoma excision in the hard palate: A preliminary study." Annals of maxillofacial surgery 7(1): 78 .

15. Keceli, H. G., B. U. Aylikci, S. Koseoglu and A. Dolgun (2015). "Evaluation of palatal donor site haemostasis and wound healing after free gingival graft surgery." Journal of clinical periodontology 42(6): 582-589. 
16. Kulkarni, M. R., B. S. Thomas, J. M. Varghese and G. S. Bhat (2014). "Platelet-rich fibrin as an adjunct to palatal wound healing after harvesting a free gingival graft: A case series." Journal of Indian Society of Periodontology 18(3): 399.

17. Ozcan, M., O. Ucak, B. Alkaya, S. Keceli, G. Seydaoglu and M. C. Haytac (2017). "Effects of Platelet-Rich Fibrin on Palatal Wound Healing After Free Gingival Graft Harvesting: A Comparative Randomized Controlled Clinical Trial." The International journal of periodontics \& restorative dentistry 37(5): e270-e278.

18. Reiser, G. M., J. F. Bruno, P. E. Mahan and L. H. Larkin (1996). "The subepithelial connective tissue graft palatal donor site: anatomic considerations for surgeons." International Journal of Periodontics \& Restorative Dentistry 16(2).

19. Rubinoff, C., E. Greener and P. Robinson (1986). "Physical properties of periodontal dressing materials." Journal of oral rehabilitation 13(6): 575-586.

20. Shakir, Q. J., P. Bhasale, N. Pailwan and D. Patil (2015). "Comparison of Effects of PRF Dressing in Wound Healing of Palatal Donor Site During Free Gingival Grafting Procedures with No Dressing at the Donor Site." J Res Adv Dent 4(1s): 69-74.

21. Singer, A. J. and R. A. Clark (1999). "Cutaneous wound healing." New England journal of medicine 341(10): 738746.

22. White, R. J. (2008). "Pain assessment and management in patients with chronic wounds." Nurs Stand 22(32): 62, 6466,68 .

23. Wynne, C. F., S. M. Ling and R. Remsburg (2000). "Comparison of pain assessment instruments in cognitively intact and cognitively impaired nursing home residents." Geriatric Nursing 21(1): 20-23.

24. Zucchelli, G., M. Mele, M. Stefanini, C. Mazzotti, M. Marzadori, L. Montebugnoli and M. De Sanctis (2010). "Patient morbidity and root coverage outcome after subepithelial connective tissue and de-epithelialized grafts: a comparative randomized-controlled clinical trial." Journal of clinical periodontology 37(8): 728-738. 